\title{
Papel del calcio en la apertura y el cierre estomático y sus interacciones con solutos compatibles. Una revisión
}

\section{Role of calcium in stomatal opening and closing and their interactions with compatible solutes. A review}

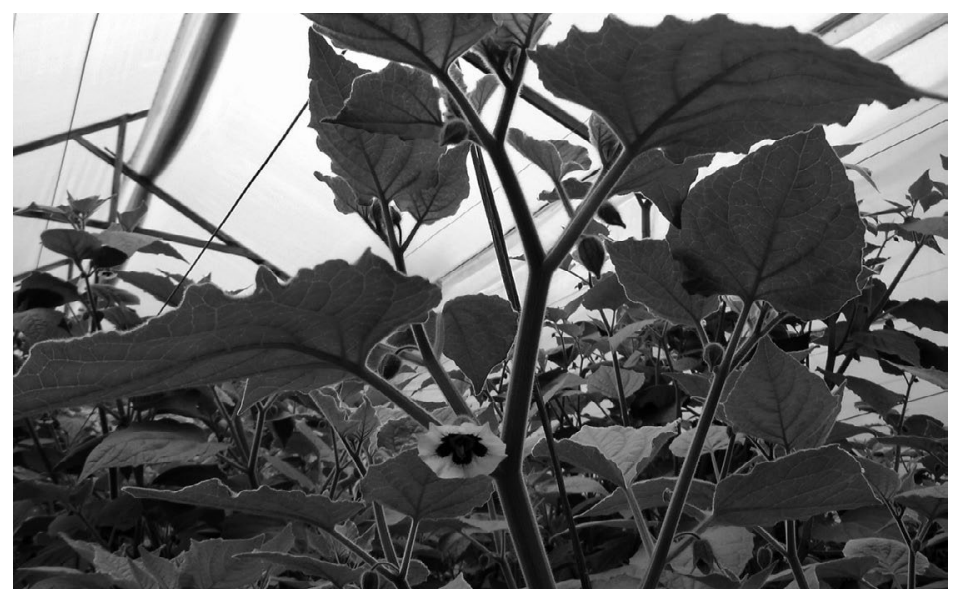

ANDREA JOHANA REYES ${ }^{1}$

JAVIER GIOVANNI ÁLVAREZ-HERRERA1,3

JUAN PABLO FERNÁNDEZ²

La regulación del intercambio gaseoso a través de los estomas, que se encuentran en su mayoría en el envés de las hojas, es crucial para el crecimiento de las plantas y los procesos de desarrollo.

Foto: G. Fischer

\section{RESUMEN}

Los canales transportan el Ca, pero es este el que está involucrado en la transducción de señales. Su apertura resulta en una disminución de la concentración de calcio citoplasmático lo cual genera variaciones espaciales y temporales de este elemento dentro de la célula, y puede llegar a causar diversas respuestas fisiológicas a diferentes estímulos bióticos y abióticos. Los canales de calcio permeables se han reportado en la membrana plasmática, tonoplasto, retículo endoplasmático, cloroplastos y las membranas nucleares de las células vegetales. El cierre de los estomas en presencia del ácido abscísico (ABA) y las actividades de los canales de calcio durante la respuesta al estrés, generan importancia a las variaciones del calcio, pues en las células guarda, las oscilaciones de $\mathrm{Ca}^{2+}$ regulan la apertura de los estomas. La sequía es uno de los mayores problemas de estrés en la agricultura. Las plantas sintetizan principalmente la hormona del estrés ABA en respuesta a la sequía, lo que provoca una cascada de señalización en las células guarda que se traduce en el cierre de los estomas, y reduce la pérdida de agua que puede influir en el uso eficiente del agua (EUA) en las plantas. Este cierre estomático involucra una relación entre el $\mathrm{Ca}^{2+}$ y el óxido nítrico ya que son intermediarios en la transducción de señales de ABA por efecto de la luz en la apertura estomática, pues es conocido que el ABA puede provocar una elevación de la concentración de $\mathrm{Ca}^{2+}$ citosólico de las células guarda, lo que ocasiona el cierre de estomas y la disminución en el proceso fotosintético.

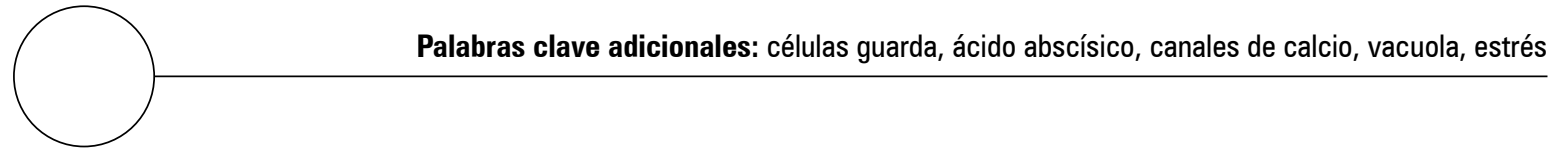

Facultad de Ciencias Agropecuarias, Grupo de Investigaciones Agrícolas, Universidad Pedagógica y Tecnológica de Colombia, Tunja (Colombia).

2 Instituto Geográfico Agustín Codazzi, Subdirección Agrología, Grupo Interno de Trabajo Levantamiento de Suelos, Bogotá (Colombia).

3 Autor para correspondencia: jgalvarezh@gmail.com 


\section{ABSTRACT}

Calcium channels are primarily involved in signal transduction. Their opening results in increased cytoplasmic calcium concentration and the spatial and temporal variations of this element within the cell, which may eventually lead to different physiological responses to various biotic and abiotic events. Calcium-permeable channels have been reported in the plasma membrane, tonoplast, endoplasmic reticulum, chloroplast and nuclear membranes of plant cells. The closing of the stomata in the presence of abscisic acid (ABA) and the activities of the calcium channel during the stress response, give importance to calcium variations because, in the guard cells, the $\mathrm{Ca}^{2+}$ oscillations regulate stomatal opening. Drought is one of the biggest stress problems in agriculture production and quality. Plants primarily synthesize the ABA stress hormone in response to drought, causing a cascade of signaling in guard cells which results in the closure of the stomata and reduces the loss of water which can affect water use efficiency in plants. This stomatal closure involves the relationship between $\mathrm{Ca}^{2+}$ and nitric oxide since they are intermediates in the signal transduction of $\mathrm{ABA}$ induced by light in the stomatal openings, because, as is known, ABA can cause an elevation of intracellular cytosolic $\mathrm{Ca}^{2+}$ in guard cells, leading to stomatal closure and reduction in the photosynthetic process.

Additional key words: guard cell, abscisic acid, calcium channels, vacuole, stress.

Los estomas son poros que se forman entre pares de células especializadas llamadas células guar$\mathrm{da}$, en las cuales se destacan dos tipos de diversidad morfológica: el primero de tipo herbáceo incluidas algunas dicotiledóneas, en este caso las células guarda presentan una hendidura entre las dos asas rodeadas por células epidérmicas, y el segundo presente en las monocotiledóneas dichas células poseen una forma arriñonada con un contorno elíptico con su poro central (Taiz y Zeiger, 2006). Estas células abren y cierran el estoma para regular el intercambio de gases entre los espacios intercelulares en el tejido de la planta y el medio ambiente circundante, lo que influye en dos de los procesos más importantes para la planta: la fotosíntesis y la transpiración (Hetherington y Woodward, 2003). El control de la apertura estomática influye directamente en los rendimientos vegetativos, así como en el control del potencial hídrico de la planta. Las células guarda se han convertido en una de las bases de la investigación y son vistas como un "modelo" para las células de las plantas terrestres. Su ubicación dentro del tejido de la hoja y la capacidad para integrar las señales ambientales y endógenas, han permitido analizar las redes de señales enlazadas al transporte de la membrana de las células guarda encargadas del control de los estomas (Hetherington y Woodward, 2003). De estos, los mecanismos de señalización inducidos por el ácido abscísico (ABA) para el inicio del cierre de estomas son los más complejos, es probable que el ABA interactúe con receptores celulares para inducir respuestas rápidas que implican alcalinización citosólica y la activación de los canales de calcio y iones de $\mathrm{K}^{+}$(Neill et al., 2003).

Los iones de calcio $\left(\mathrm{Ca}^{2+}\right)$ son importantes mensajeros secundarios de las acciones de las hormonas y de factores ambientales, en los que se 
incluyen el estrés biótico y abiótico en plantas superiores. Además, el $\mathrm{Ca}^{2+}$ está implicado en la regulación de procesos fundamentales como el tigmotropismo, gravitropismo, división celular, elongación celular, diferenciación celular, polaridad celular, fotomorfogénesis, defensa de las plantas y demás respuestas al estrés (Shao et al., 2008). La entrada de calcio y el aumento de este en el citoplasma son importantes para la transducción de señales de ABA en las células guarda (Miles et al., 2004; Covington y Harmer, 2007).

Por tanto, el objetivo de la presente revisión es describir el papel del calcio en la apertura y cierre estomático y sus interacciones con solutos compatibles, así como también determinar las consecuencias de algunos tipos de estrés abióticos y su impacto específico en la regulación y el comportamiento de las células guarda.

\section{SEÑALES QUE REGULAN LA ACTIVIDAD ESTOMÁTICA}

La regulación del intercambio gaseoso es crucial para el crecimiento de las plantas y los procesos de desarrollo, por lo tanto, las células guarda deben ser capaces de captar e integrar múltiples estímulos internos (químicos) y externos (ambientales) (Assmann y Shimazaki, 1999; Sánchez et al., 2013), entre ellos la luz, que es una de las señales ambientales dominantes del control de los movimientos estomáticos (Taiz y Zeiger, 2010). La luz azul activa la H-ATPasa de la membrana plasmática y crea un potencial eléctrico negativo intracelular a través de la membrana de las células guarda (Lee, 2010). Este flujo de salida de protones hiperpolariza la membrana plasmática, activando los canales de $\mathrm{K}^{+}$siendo este uno de los eventos que aumenta la turgencia de la célula guarda y el tamaño del poro estomático (Assmann, 1993). Bajo condiciones de estrés hídrico, el ABA se acumula en las células guarda induciendo el cierre de estomas, lo cual inhibe la apertura estomática inducida por la luz azul.
Las oscilaciones de la señal de $\left(\left[\mathrm{Ca}^{2+}\right] \mathrm{i}\right)$ pueden determinar la eficacia con la que se induce su respuesta (Cosgrove y Hedrich, 1991; Covington y Harmer, 2007; Desikan et al., 2002). En las células guarda, las oscilaciones de $\mathrm{Ca}^{2+}$ regulan la apertura de los estomas. Se ha demostrado experimentalmente que el cierre estomático no solo depende de la frecuencia y la amplitud de las oscilaciones de $\mathrm{Ca}^{2+}$, sino también en gran medida de la duración y el número de picos de $\mathrm{Ca}^{2+}$ (Allen et al., 2001; White, 2000). En particular, la larga duración del cierre estomático depende de la cantidad de picos de $\mathrm{Ca}^{2+}$. Un ejemplo de esto, es que durante el recorrido de los cinco picos de $\mathrm{Ca}^{2}$, las células guarda de Vicia faba alcanzan su máxima apertura en la mitad del tiempo (Li et al., 2004; Yangk et al., 2004).

El cierre estable de los estomas a largo plazo no solo depende de la frecuencia y la amplitud de las oscilaciones de $\mathrm{Ca}^{2+}$, sino también en gran medida de la duración y el número de picos de la concentración de $\mathrm{Ca}^{2+}$ (Marhl et al., 2006). Para los sistemas biológicos, las señales más cortas parecen poseer ventaja porque tienen un tiempo eficiente y requieren menos consumo de energía. Hay muchas evidencias experimentales sobre el tiempo límite de duración de las señales de $\mathrm{Ca}^{2+}$. Aunque los procesos biológicos de transducción de señales son siempre limitados en el tiempo, en algunos casos las señales oscilatorias son realmente muy cortas (Marhl et al., 2006).

La red de vías de señales de transducción cuenta con diversos componentes de señalización, y un pequeño número de componentes altamente interconectados que son centrales para el funcionamiento de la red (Hetherington y Woodward, 2003). El $\mathrm{Ca}^{2+}$ ha sido identificado como un componente que genera gran cantidad de interconexiones (Hetherington y Woodward, 2003). El aumento del calcio citosólico ([Ca $\left.\left.{ }^{2+}\right] \mathrm{cit}\right)$ no solo es una señal común para la mayoría de los estímulos detectados por las células guarda, sino que también es un componente común en las vías de señalización que inducen el cierre de los 
estomas y la inhibición de la apertura de los mismos (Marhl et al., 2006; Schroeder et al., 2001). Además del $\mathrm{Ca}^{2+}$, la complejidad de la señalización en la red de las células guarda requiere la participación de casi todos los mensajeros secundarios (Assmann y Shimazaki, 1999; Hetherington, 2001).

El óxido nítrico (NO) activa las defensas antioxidantes durante el estrés oxidativo y se menciona que existe una relación estrecha entre el NO y el ABA la cual aún es discutible (Neill et al., 2008). Garcia-Mata y Lamattina (2001) afirman que el $\mathrm{NO}$ participa en esta red de señalización. A partir de ese reporte, el NO y su papel principal se han convertido en un tema importante en la biología de las células guarda (Desikan et al., 2004; Nakashima y Yamaguchi-Shinozaki, 2006). Como resultado, ahora se sabe que en las células guarda el NO puede ser sintetizado por la nitrato reductasa (NR) (Desikan et al., 2004), como por la óxido-nítrico-sintasa (NOS) (Neill et al., 2004). Se reportó que el $\mathrm{NO}$ es un componente clave de la inducción dependiente de ABA en el cierre de los estomas (Garcia-Mata y Lamattina, 2007) a través de la regulación de las entradas de los canales de potasio $\left(\mathrm{K}^{+}\right)$(García-Mata et al., 2003). Aún así, el NO no ha sido reportado en el cierre de estomas inducido en ausencia de luz (Desikan et al., 2004; Gelli y Blumwald, 1997), lo cual es impedido por un inhibidor NOS (She et al., 2004). Sin embargo, solo Zhang et al. (2007) sugieren que el $\mathrm{NO}$ actúa como un intermediario de la transducción de señales de ABA para el cierre de los estomas.

La aplicación exógena de $\mathrm{Ca}^{2+}$ en Commelina communis y Arabidopsis thaliana inhibe la apertura de estomas inducida por la luz (Shao et al., 2008; White, 2000). Zhang et al. (2007) encontraron que concentraciones entre 1 y $1.000 \mathrm{mM}$ de $\mathrm{CaCl}_{2}$ pueden inhibir la apertura estomática inducida por la luz en bandas de la epidermis de $V$. faba. De acuerdo con García-Mata y Lamattina (2007) la luz no inhibe la apertura es- tomática en las bandas epidérmicas de $V$. faba, por lo que el NOS dependiente de la producción de $\mathrm{NO}$ es requerido por ABA para la inhibición de la apertura de estomas, y la aparición de una interacción $\mathrm{NO} / \mathrm{Ca}^{+}$es crítica e indispensable para la inhibición de los procesos de apertura de los estomas. Zhang et al. (2007) mencionan que tanto el $\mathrm{H}_{2} \mathrm{O}_{2}$ como el $\mathrm{NO}$, inhiben la activación de luz azul inductora de $\mathrm{H}^{+}$-ATPasa mediante la inhibición de los componentes de señalización de $\mathrm{H}^{+}$-ATPasa. Es claro entonces, que la respuesta de las células guarda es dependiente de la señalización a través de las rutas que involucran ABA y NO, pero puede estar influenciada por muchos otros factores, los cuales incluyen la concentración endógena de hormonas como etileno y metil-jasmonato y también de fuentes exógenas como el $\mathrm{H}_{2} \mathrm{~S}$ (Hancock et al., 2011), el cual remueve el $\mathrm{NO}$ de los estomas (Lisjak et al., 2010).

\section{CANALES DE CALCIO EN LAS PLANTAS}

La transducción de las señales de calcio es el mecanismo central por el cual las plantas detectan y responden a los estímulos endógenos y ambientales. La elevación citosólica de $\mathrm{Ca}^{2+}$ se logra a través de dos vías celulares, el influjo de $\mathrm{Ca}^{2+}$ a través de canales en la membrana plasmática y la liberación de $\mathrm{Ca}^{2+}$ intracelular (Jammes et al., 2011), los mismos autores describen que los canales de calcio funcionan en diversas respuestas celulares, incluyendo la respuesta hormonal, la interacción planta-patógeno, el desarrollo, la simbiosis, el estrés salino y la señalización de la luz.

En general, se acepta que un rápido incremento en la concentración de calcio en el citosol está mediado por los canales de calcio que se encuentran en la membrana plasmática y las endomembranas, como la membrana vacuolar y las membranas del retículo endoplasmático (Cvetkovska et al., 2005; Miles et al., 2004). Las mediciones de $\mathrm{Ca}^{2+}$ con tintes indicadores han mostrado que el ABA puede provocar una ele- 
vación de la concentración de $\mathrm{Ca}^{2+}$ citosólico de las células guarda. Una característica adicional del ABA en los canales de $\mathrm{Ca}^{2+}$ es que puede activarse y desactivarse varias veces dentro de la célula, dando lugar a elevaciones transitorias de la concentración de $\mathrm{Ca}^{2+}$ citosólico.

Los estudios electrofisiológicos han mostrado que las plantas tienen canales de $\mathrm{Ca}^{2+}$ con diferentes tipos de mecanismos de sincronización: ligantes, de tensión y de estiramiento activo (Kohler et al., 2003; Lee et al., 2005). Sin embargo, solo un número limitado de genes que codifican los canales de $\mathrm{Ca}^{2+}$ se han aislado y expresado funcionalmente.

Las señales que determinan las concentraciones de calcio incluyen principalmente los canales de iones de calcio permeable, $\mathrm{Ca}^{2+} / \mathrm{H}^{+}$y ATPasas de calcio. Se puede pensar que los canales permeables de calcio y $\mathrm{Ca}^{2+} / \mathrm{H}^{+}$, también pueden estar implicados en la resistencia de la planta al estrés como lo hacen las $\mathrm{Ca}^{2+}$-ATPasas. Los canales de calcio se han investigado con enfoques electrofisiológicos, bioquímicos y moleculares. Se sabe que en las células guarda, la hiperpolarización de la membrana está directamente asociada con la elevación de $\left[\mathrm{Ca}^{2+}\right]$ citosólico, que sigue a la aplicación de ABA (Yang et al., 2006). Los patrones específicos del aumento de $\mathrm{Ca}^{2+}$ pueden ser también debido a que participa en el control de la respuesta de cierre de estomas (Lee et al., 2005).

Como una característica fisiológica de gran importancia en relación con la resistencia de la planta a la sequía y su rendimiento, toma vital importancia el manejo de la eficiencia en el uso del agua (EUA) (Wang et al., 2005). La investigación molecular en relación con la mejora de la EUA desempeña una parte importante en la selección del cultivo y de las variedades resistentes o tolerantes a la sequía. La tolerancia a la sequía, la productividad de la biomasa y la EUA se consideran características agronómicas importantes, las células guarda representan el tipo de células vegetales mejor caracterizadas en lo que respecta al transporte de iones y de transducción de señales.

Los canales iónicos son proteínas que median la energía lo que disminuye el flujo de iones mediante el movimiento de ellos y a través de un poro proteico regulado. Un canal de $\mathrm{Ca}^{2+}$ permeable tiene por función fisiológica mediar la entrada de $\mathrm{Ca}^{2+}$ desde el apoplasto hacia el citoplasma. Estudios en tabaco muestran la importancia de la localización celular de los canales iónicos en la determinación de la especificidad del estímulo ya que el cierre de estomas es mediado por $\mathrm{Ca}^{2+}$. Mediciones recientes de la concentración citosólica de $\mathrm{Ca}^{2+}$ en protoplastos de fríjol indican una caída dramática aunque reversible de hasta un 17\% del valor inicial, después de una breve incubación en una solución que contiene EGTA; el $\mathrm{Ca}^{2+}$ y el ABA presentan un efecto sinérgico en la inhibición de la apertura de los estomas (Schwartz et al., 1988). Por el contrario, el EGTA o los bloqueadores de los canales de $\mathrm{Ca}^{2+}$, reducen la sensibilidad de las células guarda al ABA, así mismo, Guo et al. (2008) mencionan que el $\mathrm{ABA}$ exógeno incrementó la concentración de $\mathrm{Ca}^{2+}$ citosólico $\left(\left[\mathrm{Ca}^{2+}\right]\right.$ cit), no obstante, una vez se aplicó EGTA y trifluoperazina (TFP) se invirtió el efecto en plántulas de maíz.

Los elementos de codificación de las señales del calcio también están involucrados en el cambio de propiedades anti-sequía de las plantas y de la EUA (Shao et al., 2008), al respecto, las proteínas cambian de conformación o actividad catalítica al unirse al $\mathrm{Ca}^{2+}$, ejemplo de esto son la calmodulina, calcineurina y cinasas de proteínas dependientes de $\mathrm{Ca}^{2+}$, las cuales permiten la percepción y transducción de señales desencadenadas por los altos niveles de $\mathrm{Ca}^{2+}$ (White y Broadley, 2003).

Además de lo anterior, los canales permeables de iones de calcio, $\mathrm{Ca}^{2+} / \mathrm{H}^{+}$y $\mathrm{Ca}^{2+}$-ATPasas regulan la EUA de la planta a través de la participación del $\mathrm{ABA}$ en el cierre de los estomas y en el 
cambio de la EUA de las plantas. Se menciona que la relación entre estos elementos de codificación de señales de calcio y el EUA en las plantas es de la siguiente forma:

Señales de transducción de $\mathrm{ABA} \rightarrow$ activación de elementos de codificación de señales de calcio $\rightarrow$ cambio de concentración de $\mathrm{Ca}^{2+}$ citosólico $\left[\mathrm{Ca}^{2+}\right]$ cit $\rightarrow$ señales de transducción de $\mathrm{Ca}^{2+}$ $\rightarrow$ cambios de la apertura estomática $\rightarrow$ cambios del EUA de la planta y propiedades de las plantas contra la sequía (Shao et al., 2008).

Mori et al. (2006) presentan las evidencias genéticas moleculares para los sensores que funcionan como transductores positivos de $\mathrm{Ca}^{2+}$ en la señalización estomática de $\mathrm{ABA}$, además señalan las funciones de los genes CPK6 y CPK3 y su importancia en la regulación estomática y definen los elementos de las señales de transducción de las células guarda en la regulación de los canales de iones en la planta. Como se muestra en la figura 1, las respuestas parciales del cierre de estomas y la regulación diferencial de los canales lentos (s) y rápidos ( $\mathrm{R}$ ) en las actividades de los mutantes CPK6 y CPK3 en células guarda, son análogos con los modelos paralelos que proponen los mecanismos de señalización en una red ramificada de señales de transducción de células guarda.

\section{LA ACTIVACIÓN DE LOS CANALES DE CALCIO REGULA EL FLUJO DE SOLUTOS}

La diferencia de concentraciones entre el citoplasma y el apoplasto y los comportamientos intracelulares justifican el uso de los canales de $\mathrm{Ca}^{2+}$ como vínculo entre los estímulos extracelulares y las respuestas intracelulares, esta ruta de señalización permite la elevación del calcio citoplasmático, lo que genera el cierre o apertura de las células guarda en respuesta a la aplicación de las hormonas reguladoras ABA e IAA (Pérez, 2007).
Se menciona que el volumen de las células guarda suele cambiar en un factor de dos o más entre el estado abierto y el cerrado del poro estomático, lo que implica una profunda reorganización de la membrana vacuolar (Ritte et al., 1999). Estos cambios en el volumen no pueden ser abarcados por la expansión lateral y compresión de la bicapa lipídica (Nakashima y Yamaguchi-Shinozaki, 2006; Roelfsema y Hedrich, 2005) y, por consiguiente, deben ir acompañados por cambios en la totalidad de la membrana, por lo que se infiere que una coordinada regulación del tráfico vesicular desde y hacia la membrana plasmática y tonoplasto está implícita en el cierre y apertura estomática.

Las tasas de flujo de soluto por medio de transportadores es más lenta que la producida a través de los flujos en los canales de iones (White, 2000). Durante la apertura estomática, el aumento del volumen de la célula guarda es dirigido por la entrada y síntesis intracelular de solutos, lo que disminuye el potencial hídrico de la célula guarda creando una fuerza impulsora para la absorción del agua en las mismas (Wu et al., 2012). Debido al refuerzo de protección de las paredes celulares, el aumento de la turgencia hace que las células guarda tiendan a separarse, de manera cada vez mayor del poro estomático. Durante el cierre de estomas, hay una reducción en el contenido de solutos y volumen en la célula guarda, lo que resulta en una disminución de la apertura de los estomas. Desde las células guarda maduras, toda la absorción de solutos y flujo de salida debe realizarse a través de los canales iónicos y transportadores de iones situados en la membrana plasmática (White, 2000).

Reportes de Niinemets et al. (2005) sobre apertura estomática mencionan que el flujo de solutos desde el citosol mediado por la $\mathrm{H}^{+}$-ATPasa hiperpolariza la membrana más allá del potencial de equilibrio para el $\mathrm{K}^{+}$y regula el voltaje activo hacia el interior de los canales de $\mathrm{K}^{+}$, lo que lleva a una afluencia de $\mathrm{K}^{+}$. La producción 
de malato a partir de la degradación del almidón proporciona las principales especies aniónicas que se acumulan durante la apertura de los estomas. La absorción mediada por transportadores de $\mathrm{Cl}^{+}$y $\mathrm{NO}^{-}$puede contribuir a la acumulación de solutos intracelulares como importadores de azucares sintetizados. Durante el cierre de estomas, la despolarización de la membrana se produce debido a la inhibición de la actividad de $\mathrm{H}^{+}$-ATPasa y la activación de los canales de aniones que median el flujo pasivo de $\mathrm{Cl}^{-}$, malato ${ }^{2-}, \mathrm{y} \mathrm{NO}_{3}^{-}$. La elevación de la concentración citosólica de $\mathrm{Ca}^{2+}$ libre $\left(\left[\mathrm{Ca}^{2+}\right]\right.$ cit) a través de los canales de $\mathrm{Ca}^{2+}$ permeables en la membrana plasmática, así como la apertura de los canales de $\mathrm{Ca}^{2+}$ situados en las endomembranas es observada con frecuencia, previo al cierre estomático.

Pendenly et al. (2007) afirman que la vacuola celular es un gran acumulador de una alta cantidad de solutos y que la regulación coordinada del flujo de solutos en la membrana plasmática y el tonoplasto es fundamental para el control en la turgencia de las células guarda. Algunos modelos que representan las proteínas esenciales de señalización y segundos mensajeros involucrados en la regulación del transporte iónico de las células guarda se muestran en la figura 2.

\section{LAS VÍAS DEL CALCIO}

Diversos estímulos extracelulares específicos provocan marcas de calcio que pueden ser reconocidos por diferentes sensores del mismo. La calmodulina ( $\mathrm{CaM}$ ), el receptor de calcio predominante, es uno de los sensores de calcio mejor caracterizadas en eucariotas. Algunas proteínas como las quinasas, son propensos a actuar como "núcleos" durante la transducción de la señal de calcio. Con ello, una mejor comprensión de la función de estas proteínas contribuiría al desciframiento de la relación $\mathrm{Ca} / \mathrm{red}$ señal mediada por calmodulina y su papel en el crecimiento de las plantas, el desarrollo y la respuesta a los estímulos ambientales (Yang y Poovaiah, 2003).

La gran diferencia de las concentraciones de $\mathrm{Ca}^{2+}$ entre el citoplasma, el apoplasto y los compartimientos intracelulares, posiblemente es la razón del uso de las señales de $\mathrm{Ca}^{2+}$ como enlace entre los estímulos extracelulares y las respuestas intracelulares, esta ruta de señalización permite la elevación del calcio citoplasmático generando la apertura y el cierre de las células guarda en respuesta a la aplicación de hormonas reguladoras ABA y IAA (Pérez, 2007). El ABA puede inhibir los canales de $\mathrm{K}^{+}$que participan en la apertura de los estomas y en el cierre de los estomas, sin provocar aumentos de calcio detectables en las células guarda (Guo et al., 2008). De acuerdo con Assmann y Wang (2001) en primer lugar, el ABA inducido también aumenta el $\mathrm{pH}$ antes del cierre de los estomas, y en segundo lugar, el pH elevado activa el canal de salida de $\mathrm{K}^{+}$de la membrana plasmática, que se produce durante el cierre de los estomas, canal que es insensible a $\mathrm{Ca}^{2+}$. Las actividades del canal rápido vacuolar, el canal lento vacuolar; $\mathrm{K}^{+}$-canal selectivo vacuolar, canal vacuolar $\mathrm{Ca}^{2+}$-dependiente del voltaje, son también estimuladas por el pH alcalino citosóli$\mathrm{co}$, aunque el canal lento vacuolar se inhibe bajo estas condiciones. Estos datos implican que los cambios producidos en función de las variaciones en el $\mathrm{pH}$ junto al $\mathrm{Ca}^{2+}$ modulan el movimiento de los estomas (Assmann y Wang, 2001).

\section{RELACIONES DE TURGENCIA DE LAS CÉLULAS GUARDA}

Los iones de calcio son mensajeros secundarios bien establecidos en las vías de transducción de señales, responsables del control de la turgencia de las células guarda. Al investigar la respuesta a la concentración del calcio extracelular $\left(\left[\mathrm{Ca}^{2+}\right]\right.$ ext), McAinsh et al. (1997) observaron que diferentes concentraciones de $\left[\mathrm{Ca}^{2+}\right]$ ext producían distintos patrones de oscilación de la $\left[\mathrm{Ca}^{2+}\right]$ cit. 
Ellos descubrieron que para la $\left[\mathrm{Ca}^{2+}\right]$ ext, y, posteriormente para el ABA es posible correlacionar la fuerza del estímulo externo tanto con el modelo de oscilación de $\left[\mathrm{Ca}^{2+}\right]$ cit y la magnitud del cierre de los estomas. También encontraron que las células guarda pueden integrar información de múltiples oscilaciones de $\left[\mathrm{Ca}^{2+}\right]$ cit que inducen estímulos $\left(\mathrm{Ca}^{2+}, \mathrm{K}^{+}\right.$, ABA y manitol externos) para formular la apropiada señal de calcio en la apertura estomática. Lo anterior sugiere que la célula guarda es capaz de leer o descifrar el patrón de intensidades de la $\left[\mathrm{Ca}^{2+}\right]$ cit (Evans et al., 2001). Del mismo modo, Yang et al., (2006) mencionan que la $\left[\mathrm{Ca}^{2+}\right]$ ext cumple un papel importante en la apertura estomática ya que regula los canales de agua, este comportamiento fue observado en (Vicia faba L.).
La incidencia y características de los estímulos inducidos por las oscilaciones de la $\left[\mathrm{Ca}^{2+}\right]$ cit, estrés osmótico y los incrementos de ABA, se han observado en muchos tipos de células diferentes, al respecto, Liu et al. (2010) menciona este estímulo en células de raíces de maíz. El conocimiento de la forma en que las oscilaciones de las $\left[\mathrm{Ca}^{2+}\right]$ cit se presentan genera una clave para entender cómo se codifica la especificidad de los sistemas de señalización con base en el calcio (Evans et al., 2001). Los mismos investigadores describen que los cambios en la tasa a la cual el calcio entra y sale del citosol puede formar la base de cualquier mecanismo que genera oscilaciones de $\left[\mathrm{Ca}^{2+}\right]$ cit. Estos incluyen los flujos de entrada y flujo de salida a través de la membrana plasmática y la liberación y captación en los depósitos intracelulares, incluida

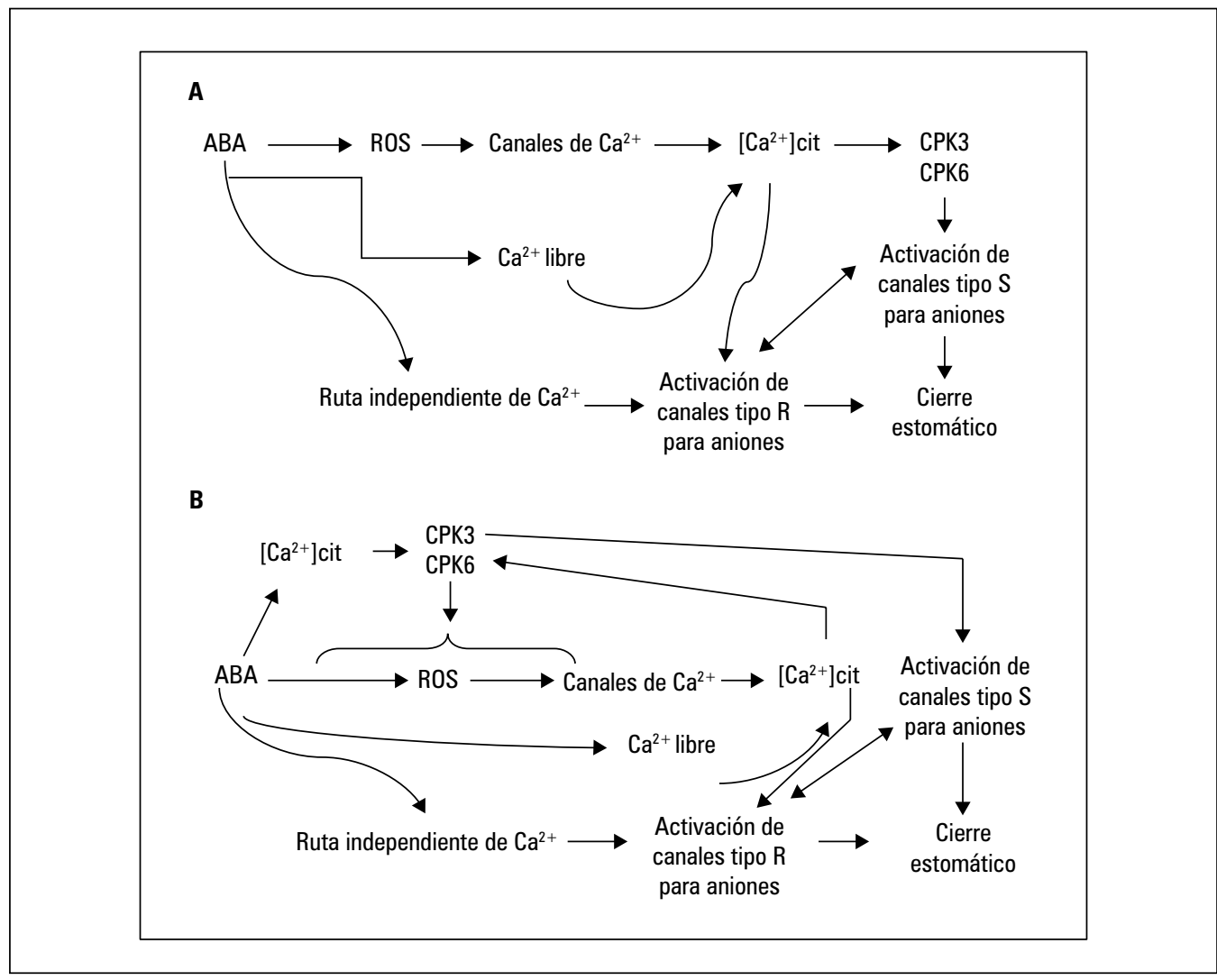

Figura 1. Modelo simplificado de transducción de señales de la red del ABA y funciones de los genes CPK3 y CPK6 en los canales de $\mathrm{Ca}^{2+}$ de la membrana plasmática de células guarda y las causas de los mecanismos intracelulares de liberación de $\mathrm{Ca}^{2+}$ citosólico. A. Ruta paralela B. Mecanismo de regulación transcripcional. Adaptado de Mori et al. (2006). 


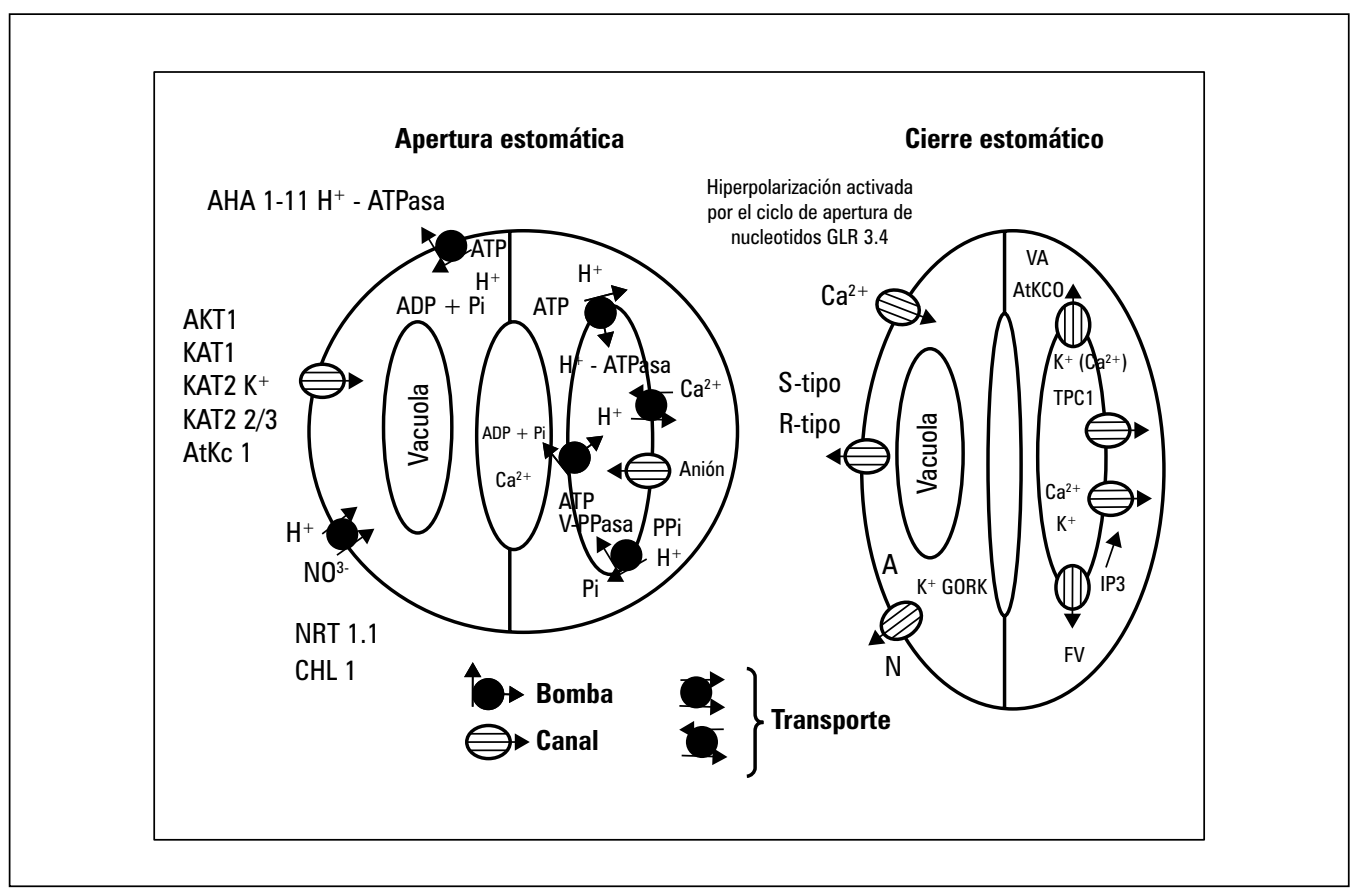

Figura 2. Canales iónicos y funcionamiento de los transportadores en los movimientos estomáticos, basado en Pendenly et al. (2007).

una contribución del núcleo. En consecuencia, es probable que las oscilaciones en la $\left[\mathrm{Ca}^{2+}\right]$ cit reflejen el funcionamiento coordinado de los canales permeables de calcio de la membrana plasmática, mensajero secundario mediado por la liberación de depósitos internos, el antiporte $\mathrm{H}^{+} / \mathrm{Ca}^{2+}$ de la membrana plasmática, las endomembranas $\mathrm{Ca}^{2+}$ ATPasa y $\mathrm{H}^{+}$-ATPasas.

\section{CONCLUSIONES}

La regulación del tamaño del poro estomático es impulsada por las variaciones de volumen de un par de células especializadas conocidas como células guarda: un aumento en la presión del volumen de las células guarda, y por lo tanto el tamaño del poro estomático. En consecuencia, una señal de estrés, reducirá la presión de turgencia celular, el volumen de las células guarda $y$, el tamaño del poro estomático, lo cual implica cambios en el funcionamiento de la planta.
Los iones de calcio son importantes mensajeros secundarios de las acciones de las hormonas y de factores ambientales, de la misma manera el $\mathrm{Ca}^{2+}$ regula los procesos como las respuestas al estrés, todo esto llevado a cabo por la entrada de calcio y el aumento de este en el citoplasma para la transducción de señales de ABA en las células guarda.

En un futuro, el proceso dinámico y en continuo crecimiento de las herramientas de investigación permitirá una compresión más completa sobre la complejidad de los eventos de señalización que regulan la respuesta estomática del $\mathrm{ABA}$, los eventos relacionados con $\mathrm{Ca}^{2+}$ y los recientes descubrimientos de su interacción con la membrana, los cuales darán una mayor visión de las funciones de la $\left[\mathrm{Ca}^{2+}\right]$ i como segundo mensajero. Del mismo modo, los estudios sobre procesos relacionados con la dinámica de la membrana pueden añadir nuevos espacios para la comprensión del transporte celular y el control del volumen y el sistema de las células guarda de las plantas superiores. 


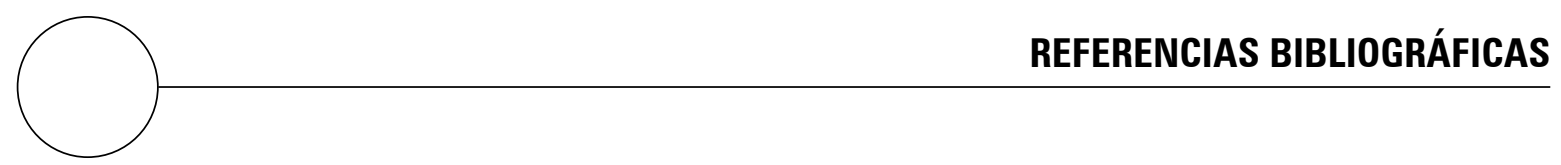

Allen, G.J., S.P. Chu, C.L. Harrington, K. Schumacher, T. Hoffman, Y. Tang, E. Grill y J. Schroeder, J.I. 2001. A defined range of guard cell calcium oscillation parameters encodes stomatal movements. Nature 411, 1053-1057.

Assmann, S.M. 1993. Signal transduction in guard cells. Annu. Rev. Cell Biol. 9, 345-375.

Assmann, S.M. y K. Shimazaki. 1999. The multisensory guard cell. Stomatal responses to blue light and abscisic acid. Plant Physiol. 119, 809-816.

Assmann, S.M. y X.Q. Wang. 2001. From milliseconds to millions of years: guard cells and environmental responses. Curr. Opin. Plant Biol. 4(5), 421-428.

Cvetkovska, M., C. Rampitsch, N. Bykova y T. Xing. 2005. Genomic analysis of MAP kinase cascades in Arabidopsis defense responses. Plant Mol. Biol. Rep. 23(4), 331-343.

Cosgrove, D.J. y R. Hedrich. 1991. Stretch-activated chloride, potassium, and calcium channels coexisting in plasma membranes of guard cells of Vicia faba L. Planta 186(1), 143-153.

Covington, M.F. y S.L. Harmer. 2007. The circadian clock regulates auxin signaling and responses in Arabidopsis. PLoS Biol. 5(8), 1773-1784.

Desikan, R., R. Graffiths, J. Hancock y S. Neill. 2002. A new role for an old enzyme: nitrate reductasemediated nitric oxide generation is required for $a b$ scisic acid-induced stomatal closure in Arabidopsis thaliana. Proc. Natl. Acad. Sci. USA. 99(25), 1631416318.

Desikan, R., M.K. Cheung, J. Bright, D. Henson, J.T Hancock y S. Neill. 2004. ABA, hydrogen peroxide and nitric oxide signaling in stomatal guard cells. J. Exp. Bot. 55(395), 205-212.

Evans, N.H., M.R. McAinsh y A.M. Hetherington. 2001. Calcium oscillations in higher plants. Curr. Opin. Plant Biol. 4(5), 415-420.

García-Mata, C. y L. Lamattina. 2001. Nitric oxide induces stomatal closure and enhances the adaptive plant responses against drought stress. Plant Physiol. 126(3), 1196-1204.

García-Mata, C. y L. Lamattina. 2002. Nitric oxide and abscisic acid cross talk in guard cells. Plant Physiol. 128(3), 790-792.
García-Mata, C. y L. Lamattina. 2007. Abscisic acid (ABA) inhibits light-induced stomatal opening through calcium- and nitric oxide-mediated signaling pathways. Nitric Oxide 17(3-4), 143-151.

García-Mata, C., R. Gay, S. Sokolovski, A. Hills, L. Lamattina y M. Blatt. 2003. Nitric oxide regulates $\mathrm{K}^{+}$and $\mathrm{Cl}^{-}$channels in guard cells through a subset of abscisic acid-evoked signaling pathways. Proc. Natl. Acad. Sci. USA. 100(19), 11116-11121.

Gelli, A. y E. Blumwald. 1997. Hyperpolarization-activated $\mathrm{Ca}^{2+}$-permeable channels in the plasma membrane of tomato cells. J. Membr. Biol. 155(1), $35-45$.

Grabov, A. y M.R. Blatt. 1998. Membrane voltage initiates $\mathrm{Ca}^{2+}$ waves and potentiates $\mathrm{Ca}^{2+}$ increases with abscisic acid in stomatal guard cells. Proc. Natl. Acad. Sci. USA 95, 4778-4783.

Guo, X.L., Y.Y. Ma, Z.H. Liu y B.H. Liu. 2008. Effects of exterior abscisic acid on calcium distribution of mesophyll cells and calcium concentration of guard cells in maize seedlings. Agr. Sci. China 7(4), 438-446.

Hancock, J.T., S.J. Neill e I.D. Wilson. 2011. Nitric oxide and $\mathrm{ABA}$ in the control of plant function. Plant Sci. $181,555-559$

Hetherington, A.M. 2001. Guard cell signaling. Cell 107(6), 711-714.

Hetherington, A.M. e I. Woodward. 2003. The role of stomata in sensing and driving environmental change. Nature 424, 901-908.

Jammes, F.J., H. Heng-Cheng, F. Villiers, R. Bouten y J. Kwak. 2011. Calcium-permeable channels in plant cells. MiniReview. FEBS Journal, 4262-4276.

Köhler, B., A. Hills y M.R. Blatt. 2003. Control of guard cell ion channels by hydrogen peroxide and abscisic acid indicates their action through alternate signaling pathways. Plant Physiol. 131, 385-388.

Lee, D., D.H. Polisensky y J. Braam. 2005. Genome-wide identification of touch-and darkness-regulated Arabidopsis genes: a focus on calmodulin-like and XTH genes. New Phytol. 165(2), 429-444.

Lee, J.S. 2010. Stomatal opening mechanism of CAM plants. J. Plant Biol. 53, 19-23 
Li, Y., G.X. Wang, M. Xin, H.M. Yang, X.J. Wu y T. Li. 2004. The parameters of guard cell calcium oscillation encodes stomatal oscillation and closure in Vicia faba. Plant Sci. 166(2), 415- 421.

Lisjak, M., N. Srivastava, T. Teklic, L. Civale, K. Lewandowski, I. Wilson, M.E. Wood, M. Whiteman y J.T. Hancock. 2010. A novel hydrogen sulfide donor causes stomatal opening and reduces nitric oxide accumulation. Plant Physiol. Biochem. 48, 931-935.

Liu, Z., Z. Ma, X. Guo, H. Shao, Q. Cui y W. Song. 2010. Changes of cytosolic $\mathrm{Ca}^{2+}$ fluorescence intensity and plasma membrane calcium channels of maize root tip cells under osmotic stress. Plant Physiol. Biochem. 48, 860-865.

Luo, X., N. Cui, Y. Zhu, L. Cao, H. Zhai, H. Cai, W. Ji, X. Wang, D. Zhu, Y. Li y X. Bai.2012. Over-expression of GsZFP1, an ABA-reponsive C2H2-type zinc finger protein lacking a OALGGH motf, reduces $A B A$ sensitivity and drecreases stomata size. J. Plant Physiol. 169, 1192-1202.

Maathuis, F.J., S.T. May, N.S. Graham, H.C. Bowen, T.C. Jelitto, P. Trimmer, M.J. Bennett, D. Sanders y P.J.White. 1998. Cell marking in Arabidopsis thaliana and its application to patch-clamp studies. Plant J. 15(6), 843-851.

MacRobbie, E. 1999. Vesicle trafficking: a role in transtonoplast ion movements?. J. Exp. Bot. 50, 925 934.

McAinsh, M.R., C. Brownlee y A.M. Hetherington. 1997. Calcium ions as second messengers in guard cell signal transduction. Physiol. Plant. 100(1), 1629.

Marhl, M., M. Perc y S. Schuster. 2006. A minimal model for decoding of time-limited $\mathrm{Ca}^{2+}$ oscillations. Biophys. Chem. 120(3), 161-167.

Marten, H., K. Konrad, P. Dietrich, M.R. Roelfsema y R. Hedrich. 2007. $\mathrm{Ca}^{2+}$-dependent and -independent abscisic acid activation of plasma membrane anion channels in guard cells of Nicotiana tabacum. Plant Physiol. 143, 28-37.

Miles, G.P., M.A. Samuel, A.M. Jones y B.E. Ellis. 2004. Mastoparan rapidly activates plant MAP kinase signaling independent of heterotrimeric $G$ proteins. Plant Physiol. 134(4), 1332-1336.

Millar, A.H., V. Mittova, G. Kiddle, J.L. Heazlewood, C.G. Bartli, F.L. Theodoulou y C.H. Foyer. 2003. Control of ascorbate synthesis by respiration and its implications for stress responses. Plant Physiol. 133, 443-447.

Mori, I.C., Y. Murata, Y. Yang, S. Munemasa, Y.F. Wang, S. Andeoli, H. Tiriac, J.M. Alonso, J.F. Harper, J.R.
Ecker, J.M Kwak y J. Schroeder. 2006. CDPKs CPK6 and CPK3 function in ABA regulation of guard cell S-type anion- and $\mathrm{Ca}^{2+}$ - permeable channels and stomatal closure. PLoS Biol. 4(10), 1749-1762.

Nakashima, K. y K. Yamaguchi-Shinozaki. 2006. Regulons involved in osmotic stress-responsive and cold stress-responsive gene expression in plants. Physiol. Plant. 126(1), 62-71.

Neill, S.J., R. Desikan, A. Clarke, R.D Hurst y J. Hancock. 2002. Hydrogen peroxide and nitric oxide as signaling molecules in plants. J. Exp. Bot. 53(372), 1237-1247.

Neill, S.J., R. Desikan, A. Clarke y J. Hancock. 2002. Nitric oxide is a novel component of abscisic acid signaling in stomatal guard cells. Plant Physiol. 128(1), 13-16.

Neill, S.J., R. Desikan y J. Hancock. 2003. Nitric oxide as a mediator of ABA signalling in stomatal guard cells. Bulg. J. Plant Physiol. Esp. 124-132.

Neill, S.J., R. Barros, J. Bright, R. Desikan, J. Hancock, J. Harrison, P. Morris, D. Ribeiro e I. Wilson. 2008. Nitric oxide, stomatal closure, and abiotic stress. J. Exp. Bot. 59(2), 165-176.

Niinemets, U., A. Cescatti, M. Rodeghiero y T. Tosens. 2005. Leaf internal diffusion conductance limits photosynthesis more strongly in older leaves of Mediterranean evergreen broad-leaved species. Plant Cell Environ. 28(129), 1552-1566.

Pandey, S., W. Zhang y S.M. Assmann. 2007. Roles of ion channels and transporters in guard cell signal transduction. FEBS Lett. 581(12), 2325-2336.

Pérez, V. 2007. Estudio de canales permeables a calcio de membranas intracelulares de células vegetales. Tesis de doctorado. Universidad de Colima, Colima, México.

Ritte, G., J. Rosenfeld, K. Rohrig y K. Raschke. 1999. Rates of sugar uptake by guard cell protoplasts of Pisum sativum L. related to the solute requirement for stomatal opening. Plant Physiol. 121(2), 647-655.

Roelfsema, M.R. y R. Hedrich. 2005. In the light of stomatal opening: new insights into 'the Watergate'. New Phytol. 167(3), 665-691.

Sánchez, C., G. Fischer y D.W. Sanjuanelo. 2013. Stomatal behavior in fruits and leaves of the purple passion fruit (Passiflora edulis Sims) and fruits and cladodes of the yellow pitaya [Hylocereus megalanthus (K. Schum. ex Vaupel) Ralf Bauer]. Agron. Colomb. 31(1), 38-47.

Schwartz, A., N. Ilan, y D.A. Grantz. 1988. Calcium efects on stomatal movement in Commelina com- 
munis L.: use of EGTA to modulate stomatal response to light, $\mathrm{KCl}$ and $\mathrm{CO}_{2}$. Plant Physiol. 87(3), 583-587.

Shao, H. B., W.Y. Song y L.Y. Chu. 2008. Advances of calcium signals involved in plant anti-drought. C.R. Biol. 331(8), 587-596.

She, X.P., X.G. Song y J.M. He. 2004. Role and relationship of nitric oxide and hydrogen peroxide in light/ dark-regulated stomatal movement in Vicia faba. Acta Bot. Sinica 46(11), 1292-1300.

Schroeder, J., G. Allen, V. Hugouvieux, J.M. Kwak, D. Waner. 2001. Guard cell signal transduction. Annu. Rev. Plant Physiol. Plant Mol. Biol. 52, 627-646

Soni, D., S. Ranjan, R. Singh, P. Khare, U. Pathre y P. Shirke. 2012. Photosynthetic characteristics and the response of stomata to enviromental determinants and ABA in Selaginella bryopteris, a resurrection spike moss species. Plant Sci. 191-192, 43-52.

Taiz, L. y E. Zeiger. 2006. Plant physiology. $3^{\text {rd }}$ ed. Sinauer Associates, Sunderland, MA.

Taiz, L. y E. Zeiger. 1998. Plant physiology. $2^{\text {nd }}$ ed. Sinauer Associates, Sunderland, MA.

Wang, J.Y., J.N. Yu, T. Chen, Z.G. Zhang, Y.J. Hao, J.S. Zhang y S.Y. Chen. 2005. Functional analysis of a putative $\mathrm{Ca}^{2+}$ channel gene TaTPC1 from wheat. J. Exp. Bot. 56(422), 3051-3060.

White, P.J. 2000. Calcium channels in higher plants. Biochimica et Biophysica Acta (BBA) - Biomembranes 1465(1-2), 171-189.
White, P.J. y M.R. Broadley. 2003. Calcium in plants. Ann. Bot. 92, 487-511.

Wu, Y. X. Liu, W. Wang, S. Zhang y B. Xu. 2012. Calcium regalates the cell to cell water flow pathway in maize roots during variable water conditions. Plant Physiol. Biochem. 58, 212-219.

Yang, H.M., X.Y. Zhang y G.X. Wang. 2004. Cytosolic calcium oscillation signaling in guard cell. Plant Sci. 166(3), 549-556.

Yang, T. y B. Poovaiah 2003. Calcium/calmodulin-mediated signal network in plants. Trends Plant Sci. 8 (10), 505-512.

Yang, H.M., X.Y. Zhang, O.L. Tang y G.X. Wang. 2006 Extacellular calcium is involved in stomatal movement through the regulation of water channels in broda bean. Plant Growth Regul. 50(1), 79-83.

Yang, J., I. Ordiz, J. Jaworski y R. Beachy. 2011. Induced accumulation of cuticular waxes enhances drought tolerance in Arabidopsis by changes in development of stomata. Plant Physiol. Biochem. 49, 1448-1455.

Zhang, X., A. Takemiya, T. Kinoshita y K. Shimazaki. 2007. Nitric oxide inhibits blue light-specific stomatal opening via abscisic acid signaling pathways in Vicia guard cells. Plant Cell Physiol. 48(5), 715 723.

Zhao, X., X. Qiao, J. Yuan, X. Ma y X. Zhang. 2012. Nitric oxide inhibits blue light-induced stomatal opening by regulating the $\mathrm{K}^{+}$influx in guard cells. Plant Sci. 184, 29-35. 